\title{
The granularity of gravity
}

\author{
Engel Roza \\ Stripperwei 1, 5551 ST Valkenswaard, The Netherlands \\ Email: engel.roza@onsbrabantnet.nl
}

\section{Summary}

It is shown that Verlinde's hypothetical concepts of entropic gravity can be successfully applied to the author's previous work on the modification of the Newtonian gravity by Einstein's Cosmological Constant. It allows an assessment by theory for Milgrom's empirical acceleration constant, thereby revealing some fundamental differences with Verlinde's conclusions.

Keywords: entropic gravity; Cosmological Constant; quantum gravity; dark matter

\section{Introduction}

This article is meant as a supplement to a previous study [1]. In particular as an extension of its discussion paragraph. It is my aim to give some view on the granularity of the gravitational space, in which I will borrow some concepts from Verlinde's "entropic gravity" [2,3,4,5]. I have decided to separate the issue from my previous article, because of the need to accept the somewhat hypothetical, if not speculative, nature of these concepts. That concepts are needed is beyond doubt, because otherwise there are no means to give an explanation for the negative pressure executed by the spatial fluid that must be present in vacuum for explaining a positive value of the Cosmological Constant in Einstein's Field Equation. Such a value is required to remove the anomaly of particular cosmological phenomena, like the stellar rotation curves in galaxies and the accelerated expansion of the universe. In my previous article, it has straightforwardly been derived that in a gravitational system with a central mass $M$ in vacuum, the Cosmological Constant, while independent of spacetime coordinates, amounts to $\Lambda=\lambda^{2} / 2=a_{0} / 5 M G$, where $a_{0}\left(\approx 10^{-10} \mathrm{~m} / \mathrm{s}^{2}\right)$ is Milgrom's acceleration constant $[6,7,8]$ and $G$ the gravitational constant.

Satisfying Einstein's Field equation in vacuum under absence of a massive source under the condition of a positive Cosmological Constant $\Lambda$ requires the presence of a background energy [9]. Under inclusion of the massive source, the background energy will of course still be there and seems to show up as polarized dipoles [1], which would explain the $1 / M$ dependency of the Cosmological Constant. It is my aim trying to harmonize the dipole modeling of the spatial fluid with Verlinde's entropic gravity concept. The first step is pointing out that this concept can be interpreted as the modeling of the spatial fluid by an elastic glassy medium and, secondly, that elasticity can be modeled in terms of dipoles.

\section{Entropic gravity}

Let me start by giving an synopsis of Verlinde's theory. This might be useful, because Verlinde's articles are written in the formalism of the string theory and are therefore hardly accessible for nonexperts (like me). Nevertheless, it will appear to be possible to extract the fundamentals and to reformulate these in a less abstract terminology. The gravitational model that I wish to describe applies to a pointlike massive source in vacuum (I prefer to use the word vacuum over empty space, because of the obvious reason that we cannot escape from the spatial fluid appearance in Einstein's Field Equation with Cosmological Constant). The massive source is an abstraction of some (baryonic) matter with a volume mass density $\rho_{B}(r)$ present within some sphere with radius $R$, such that $\rho_{B}(r)=0$ for $r \geq R$. This baryonic mass executes a gravitational force $F_{N}$ on test particles with 
mass $m$ beyond the sphere, which in the weak field limit of Einstein's equation coincides with the Newtonian law that at the higher abstraction level is derived from Poisson's equation,

$$
\begin{aligned}
& \frac{1}{r^{2}} \frac{d}{d r}\left(r^{2} \frac{d \Phi}{d r}\right)=-4 \pi G \rho_{B}(r) \rightarrow r^{2} \frac{d \Phi}{d r}=-G \int_{0}^{R} \rho_{B}(r) 4 \pi r^{2} d r, \text { such that } \\
& F_{N}=-m \frac{d \Phi}{d r}=m \frac{G}{r^{2}} \int_{0}^{R} \rho_{B}(r) 4 \pi r^{2} d r .
\end{aligned}
$$

Beyond the radius of the sphere, the mass distribution within the sphere is irrelevant. This implies that the value of the integral can simply be indicated as the encapsulated baryonic mass $M$. Even so, if desired, one might replace the volume mass distribution by an equivalent surface mass distribution $\sigma_{D}(r)$ on the shell of the sphere. The ultimate abstraction is, to model the encapsulated baryonic mass as a pointlike massive source that can be modelled as a Schwarzschild black hole with mass $M$ and a radius $R_{S}=2 M G / c^{2}$, where $c$ is the speed of light in vacuum.

Doing so, the properties of the black hole will appear on the shell of the sphere that encapsulates the baryonic mass. This view is known as "the holographic principle". One of these properties is the entropy of the black hole (hence the entropy of the encapsulated mass). The origin for assigning this property is the question of how many molecules are contained within the radius of a black hole. The answer is: as many as degrees of freedom for the energy of the molecules are available. The (dimensionless) informatics entropy $S$ is related with the logarithm of this number. Bekenstein [10] theorized that

$$
S=\frac{A c^{3}}{4 G \hbar}, \text { where } A=4 \pi R_{S}^{2}
$$

and Hawking [11] subsequently argued that entropy should go hand in hand with temperature. He found,

$$
T_{H}=\frac{\hbar c^{3}}{8 \pi G M k_{B}} .
$$

In fact, he derived this result by a similar approach as Unruh [12] derived for the temperature experienced by a detector at constant acceleration $\mathbf{a}$ in vacuum as

$$
k_{B} T=\frac{1}{2 \pi} \frac{\hbar|\mathbf{a}|}{c} .
$$

Unruh's result coincides with Hawking's result if $\mathbf{a}$ is taken as the Newtonian acceleration at the black hole's radius $R_{S}$, i.e., if

$$
|\mathbf{a}|=\frac{M G}{R_{S}^{2}}
$$


In these expressions $\hbar$ and $k_{B}$, respectively are Planck's (reduced) constant and Boltzmann's constant.

In his entropic gravity concept, Verlinde heuristically associates these properties with the Newtonian gravity. He does so by hypothesizing an amount of $N=4 S$ virtual molecules on the holographic screen and assigning an energy $k_{B} T / 2$ to each of these. To me, it makes more sense to adopt $N=S$ in conjunction with $2 k T$ instead. Initially, it will give the same result. Later in this article it will be shown why this second option, apart from its rationale, is the better one. In both cases, it enables identifying the energy equivalent $M^{\prime}\left(=M c^{2}\right)$ of the encapsulated mass as,

$$
M^{\prime}=2 N k_{B} T=4 \frac{A c^{3}}{G \hbar} k_{B} T
$$

Subsequently eliminating $N$ from (3) and (7), gives,

$$
T=\frac{M G \hbar}{2 \pi k_{B} c} \frac{c^{4}}{4 M^{2} G^{2}}=\frac{\hbar c^{3}}{8 \pi G M k_{B}} .
$$

Curiously, this just gives the Hawking temperature! To associate these concepts with gravity, Verlinde states that, as soon as a massive test particle approaches the holographic screen at a distance equal to its (reduced) Compton wave length, it will be unified with the virtual molecules on the screen. This unification takes place under influence of a force derived from the second law of thermodynamics, which subsequently is identified as the gravitational force. Quantitatively, this process is analyzed as follows. From thermodynamics we have the increase of (non-dimensionless) physical entropy by an amount $\Delta S$ under supplying an energy amount $\Delta E$, such that

$\Delta E=T \Delta S$

The unification of a test particle wit a (reduced) Compton wave length $\Delta x=\hbar / m c$, is equivalent with a supplied energy $\Delta E=F \Delta x$, where $F$ is the force that displaces the test particle over a distance $\Delta x$. Verlinde attributes the origin of the unifying force from the temperature of the holographic screen by reversing Unruh's argumentation, by stating that the force is the result of the screen's temperature, rather than the opposite. Hence,

$$
\Delta E=F \frac{\hbar}{m c}
$$

where from (5),

$$
F=2 \pi k_{B} T \frac{m c}{\hbar}
$$

From (9) , (10) and (11) we get,

$$
\Delta E=2 \pi k_{B} T=T \Delta S,
$$

such that the change in entropy as a consequence of the unification appears being, 
$\Delta S=2 \pi k_{B}$

From (11) and (6), and considering that Poisson's law allows a transport of the mass properties of the black hole (with radius $R_{S}$ ) to the screen that encapsulates the massive sources (with radius $r$ ), it follows readily,

$F=\frac{m M G}{r^{2}}$

in which we recognize Newton's law.

This is an interesting result, because it gives an entropic view on gravity. However, it is not emergent in the sense that it is derived from "first principles". The reason is the presence of a loop hole in the derivation. Basically, the derivation relies upon Poisson's equation, which says that the mass distribution of encapsulated baryonic mass is irrelevant for the force that is experienced for a test particle beyond the encapsulation. Moreover, Hawking's temperature expression (4) relies on the application of the Newtonian law, which can be seen from replacing the generic acceleration $\mathbf{a}$ in Unruh's expression by the Newtonian $g$ at the radius $R_{S}=2 M G / c^{2}$. It is therefore not surprising that Newton's law pops up, because it was already there. Obviously, Verlinde's derivation contains some heuristic assumptions and numerical fixings, such as a logarithmic measure $N=4 S$ virtual particles in conjunction with an energy $k T / 2$ (or, equivalently, $N=S$ in conjunction with $2 k T$ ), and the reduced Compton wave length for the unification. Nevertheless, this entropic view is a challenging picture of gravity, which might give a clue for solving outstanding problems.

The successful generalization of the entropy of a black hole to the entropy of encapsulated mass gives a lead to the idea for conceiving mass as a manifestation of entropy. One might even play, like Verlinde does, with the idea that entropy is the cradle of matter and that conversion from entropy into matter would be possible. Inspired by this, Verlinde proposes a modification of the Newtonian gravity, which could possibly give an explanation for the unsolved gravitational problems. In this theory, the holographic screen (short for the screen that encapsulates baryonic matter), does not longer solely possess the entropy properties of a black hole. Instead, the entropy is built up by the entropy of a black hole (with mass equal to the mass of the encapsulated matter) and an additional entropy subtracted from the vacuum between the black hole and the cosmological horizon. This implies that Verlinde hypothesizes that, in spite of the absence of matter, entropy can be assigned to the vacuum. By generalizing Bekenstein's entropy of a (massive) black hole $S$ as given by (3), Verlinde assigns an entropy $S_{D}(r)$ to a (mass less) sphere in vacuum with radius $r$, to the amount of

$$
S_{D}(r)=\frac{r}{L} \frac{c^{3} A(r)}{4 G \hbar}
$$

where $L$ is the cosmological horizon. Subsequently, Verlinde hypothesizes that baryonic matter put into the vacuum subtracts entropy from the vacuum. The subtracted entropy has an energy equivalent (and therefore a mass equivalent). The subtracted energy is beneficial to the baryonic matter, which, as a consequence, is virtually increased. Because change of entropy implies a change of volume, the vacuum volume shrinks. Because such a shrink is not without a resistance, an amount of elastic energy is involved. Therefore, it must be possible to calculate the virtual increase of baryonic mass from the elastic energy from the volume shrink caused by putting matter into the vacuum. From this point we could try to follow Verlinde's analysis. Let me admit that I am unable to 
do so. Let me therefore try to harmonize the dipole view $[13,1]$ straight from this point. This needs a re-interpretation of the vacuum's entropy.

\section{Entropy of the vacuum}

For a better understanding of the relationship between the vacuum's entropy and the entropy of a black hole, it might be instructive to visualize the entropy of a black hole in a way as suggested by Susskind [15]. He proposed to consider the black hole's mass $M$ as a sum of $N$ elementary amounts $\Delta M$, brought in or radiated off, by bosons. To this end, the (reduced) Compton wavelength of $\Delta M$ must equate the periphery $2 \pi R_{S}$ of the black hole, such that

$$
\begin{aligned}
& 2 \pi R_{S}=\frac{\hbar}{c \Delta M} \rightarrow \Delta M=\frac{\hbar}{2 c \pi R_{S}} \rightarrow \\
& S=N=\frac{M}{\Delta M}=\frac{2 c \pi R_{S}}{\hbar} M=\frac{2 c \pi R_{S}}{\hbar}\left(\frac{2 M G}{c^{2}}\right) \frac{c^{2}}{2 G}=\frac{\pi c^{3}}{\hbar G} R_{S}^{2}=\frac{c^{3}}{4 \hbar G} A .
\end{aligned}
$$

The result of this simplistic view nicely corresponds with the results of the rigid analysis as originally performed by Hawking [11] and repeated by others [16]. These analyses give the correct answer to the question how to handle the Compton wavelength (reduced or non reduced) and on the correct value of the numerical proportionality factor related with the area $A$ (which was unknown in Bekenstein's conceptual set up). Where the entropy expression for the black hole is an area law, Verlinde's entropy expression (15) for the vacuum basically is a volume law. Rather than counting the number of elementary massive amounts $\Delta M$ that are actual present, it counts the number of elementary massive amounts $\Delta M$ that can be maximally comprised by the vacuum. This maximum is bound by the "emerging" area of the cosmological horizon. The amount of these virtual masses comprised by the holographic screen that encapsulates the baryonic mass responsible for the gravitational force, is the $r / L$ fraction of the maximum. The area law gives expression to an information bound, while the volume law gives expression to the way how the information is organized. Entropy is a measure of information, not more, not less. Information presupposes a carrier, being signals or circuits, like in Shannon's case, or massive energies, like in Bekenstein's case. Verlinde's entropy assignment to the vacuum, implicitly presupposes a carrier as well. To be meaningful, the entropic virtual masses must represent some mass less physical energetic entities. It is a straightforward step to conceive the entropic virtual masses as omni-present dipoles, which, under influence of the baryonic gravitational force, are polarized within the cosmological screen.

\section{Dipole interpretation}

Rather than assuming $N$ massive particles on the holographic screen, we assume $N$ dipoles, like illustrated in figure 1. If there is a reason why a particle beyond the holographic screen unifies with the screen under influence of an entropic force triggered by the screen's temperature, there will be no reason why a particle within the screen should not do the same. It leaves, so to say, a "hole" in the encapsulated mass. The dipole has a positive energy kernel and a negative energy kernel, but the massive content is zero. According to the theory as described before, the spacing will be two (reduced) Compton lengths. We proceed now as follows. Eqs. (15) and (3) allow to establish the number of dipoles $N$ that cross the holographic screen, because

$$
N=S=\frac{r}{L} \frac{c^{3} A(r)}{4 G \hbar}
$$

The strength $p_{d}$ of a dipole, consisting of elementary kernels of energy with mass equivalent $m_{d}$ and spaced by two reduced Compton lengths $d=2 \hbar / m_{d} c$, amounts to 
$p_{d}=m_{d} d=2 \frac{\hbar}{c}$

The difference $\Delta N$ between the amount of dipoles on the holographic screen and the screen shifted by an amount $\Delta r$ amounts to

$$
\Delta N=\frac{r}{L}\left\{\frac{c^{3} A(r+\Delta r)}{4 G \hbar}-\frac{c^{3} A(r)}{4 G \hbar}\right\}=\frac{r}{L} \frac{c^{3}}{4 G \hbar}\left\{4 \pi(r+\Delta r)^{2}-4 \pi r^{2}\right)=2 \pi \frac{r^{2}}{L} \frac{c^{3}}{G \hbar} \Delta r .
$$

The volume difference $\Delta V$ between the two screens is,

$$
\Delta V=\frac{4}{3} \pi(r+\Delta r)^{3}-\frac{4}{3} \pi r^{3} \approx 4 \pi r^{2} \Delta r
$$

The dipole moment density $P_{g 0}$ therefore amounts to

$$
P_{g}=\frac{p_{d} \Delta N}{\Delta V}=2 \frac{\hbar}{c} \frac{c^{3}}{G \hbar} \frac{2 \pi r^{2}}{4 \pi r^{2} L}=\frac{c^{2}}{G L}
$$
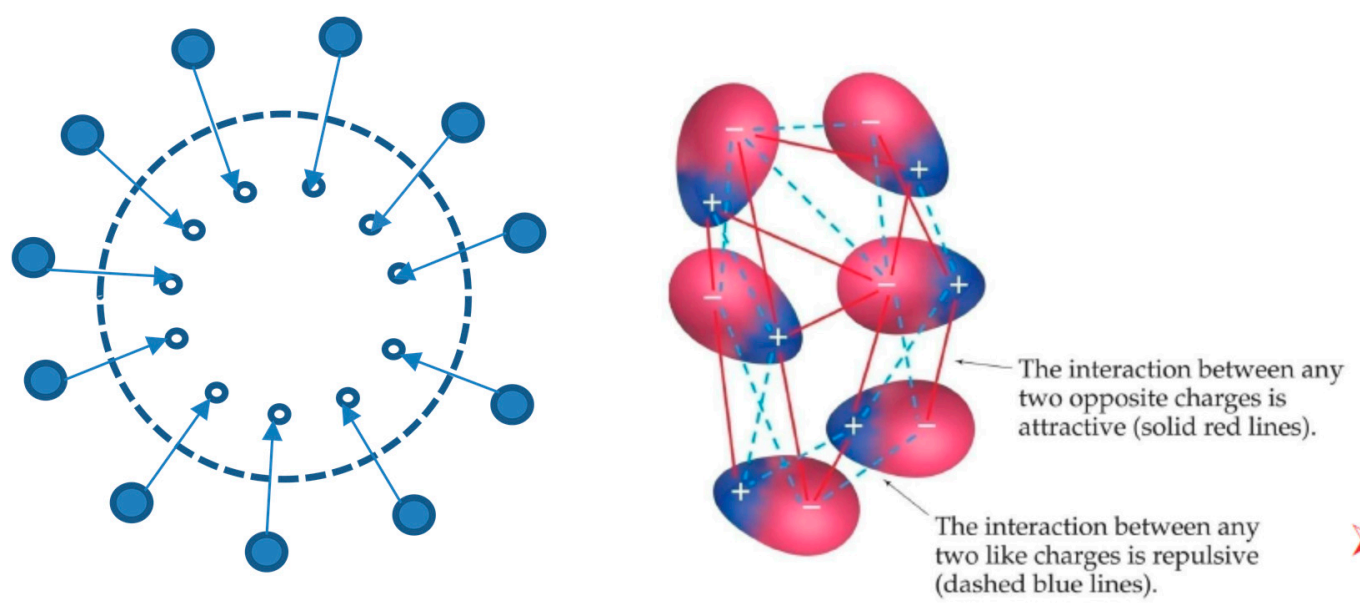

Fig.1. Left: Modelling the entropy of the vacuum as dipoles across the holographic screens up to the cosmological screen. Right: dipole-dipole interaction in an elastic medium (from:T.L. Brown, H.E. LeMay, B.E. Bursten, Chemistry, The Central Science, $10^{\text {th }}$. Ed, Chapter 10).

Replacing the cosmological scale $L$ (Hubble length) by the acceleration scale $a_{L}=c^{2} / L$, we have

$$
P_{g}=\frac{a_{L}}{G}
$$

This result allows a comparison with the dipole modelling of the Cosmological Constant, which resulted into [1],

$$
P_{g 0}=\frac{a_{0}}{20 \pi G}
$$


where $a_{0}$ is Milgrom's acceleration constant. Equating (22) with (23) allows calculating this constant from theory as,

$a_{0}=20 \pi a_{L}$.

Equating the Hubble length as $L=c t_{L}$ and $t_{L} \approx 13.5$ Gyear, we get from (24),

$a_{0}=1.2 \times 10^{-10} \mathrm{~m} / \mathrm{s}^{2}$.

This is the very same value as Milgrom's empirical one [13].

\section{Comparison}

Verlinde's conclusions are somewhat different. Instead of (24), Verlinde concludes,

$a_{0}=a_{L} / 6$.

This is substantially different and less close to Milgrom's value. Moreover, Verlinde modifies the gravitational acceleration constant to

$g(r)=g_{N}(r)+\sqrt{a_{0} g_{N}(r)}$,

where $g_{N}(r)$ is the Newtonian one.

Verlinde claims a match of this result with Milgrom's hypothesis, which is confirmed by a wealth of cosmological observations on galaxies. To my opinion Verlinde's conclusion here is incorrect. Milgrom's equation is different, nl.,

$g=\frac{g_{N}}{\mu(x)}$, with $x=g / a_{0}$ where $\mu(x)=\frac{x}{\sqrt{1+x^{2}}}$.

This implies that at small $r$ Milgrom's gravitational acceleration coincides with the Newtonian one. This is not true for (27). Probably, the difference is sufficiently significant for being checked by observations. The author's result, on the other hand, coincides for small $r$ with the Newtonian one, but is different from Milgrom's one at extreme cosmological distance.

In view of this, it seems to be fair giving support to Hajdukovic's view [14] that the vacuum is filled by a fluid consisting of grains that are structured as confined dipoles with a matter kernel and an antimatter kernel, spaced by twice their Compton wave lengths.

\section{The size of the grains}

The volume size $V_{g}$ of the dipole grains follows readily from (19) and (20) as

$V_{g}=\frac{\Delta V}{\Delta N}=2 \frac{G \hbar L}{c^{3}}$

Assuming that the vacuum shows a packing density $\kappa$ of spherical grains with radius $R_{g}$, we get 
$R_{g}=\left(\kappa \frac{3 G \hbar L}{2 \pi c^{3}}\right)^{1 / 3}$

Equating $\kappa$ with the optimum spherical packing density (Kepler's conjecture)

$\kappa=\pi /(3 \sqrt{2})$

and considering that $L=c t_{L}\left(t_{L}=13.5\right.$ Gyear $), R_{g}$ is calculated as,

$R_{g}=1.6 \times 10^{-14} \mathrm{~m}$.

Each of these grains contains a gravitational dipole with strength $2 \hbar / c$. The right-hand part of figure 1 illustrates that they assemble an elastic medium. In absence of a gravitational force their orientation is randomly directed. The polarization has a similar effect as a displacement charge that enhances the electric field strength in a dielectricum.

It is tempting to relate the grain size and the dipole strength with mass values attributed to the two poles. At this stage, I prefer not doing so. It has to do with the suggestive relationship between the gravitational dipole and the nuclear mesons. Both type of constructs are configurations of a kernel with positive energy and a kernel of negative energy in an equilibrium of a repelling force (in particle physics due to Yukawa's $\exp (-\lambda r) / \lambda r$ potential) and an attracting confinement force (in particle physics the gluing colour force). In this respect, I would like to point to an intriguing observation. In a previous work, I have expressed the gravitational constant $G$ in quantum mechanical quantities [17], which probably so far is just considered as a curiosity, because of an unconventional view on the binding force between quarks. The expression contains a factor $\alpha$ of order 1 , which had to be set as $\alpha=0.69$, to obtain a perfect fit between the derived expression and the numerical value of $G$. In that work, the factor has been introduced as a correction factor on the spacing between the two quarks built up as a standing wave with a half non-reduced Compton wave length. So far, it has troubled me why on the one hand the result of the theory has been so close to the empirical value of $G$, while on the other hand I needed the correction factor. Curiously, the theory described in the present article gives the answer. It says that the spacing in the dipole has to be set at $2 x$ the reduced Compton wave length, which gives about the same value $(2 \times 1 / 2 \pi \approx 0.69 \times 1 / 2)$. More research is needed to put these correspondences in perspective.

As a final remark I would like to note that, in spite of the fact that the concept of temperature has been leading to the entropic vision, in retrospect it does not play an essential role. In a way, this is satisfying, because it enables to maintain temperature as a macrostate of molecules and gravity as a force with an origin at a baryonic level below the molecular one.

\section{Conclusion}

Interpreting Verlinde's vacuum entropy as quantum mechanical bosons allows conceiving the vacuum as a fluid of gravitational dipoles subject to polarization under influence of a baryonic source. Consequently, the vacuum behaves similarly as the gravitational equivalent of a dielectrum showing the equivalent of a displacement charge, thereby effectively enhancing the strength of the baryonic source.

\section{References}

[1] E. Roza, doi:10.20944/preprints201705.0164.v3 (2017) 
[2] E. Verlinde, JHEP 1104, 029 ; arXiv: 1001.0785v1 [hep-th] (2011)

[3] E. Verlinde, arXiv:1611.02269v2 [hep-th] (2016)

[4] A. McCoss, Journ. of Quantum Inf. Sc, 7, 67 (2017)

[5] M.M. Brouwer et al., Monthly Notices Royal Astronomical Soc., Vol. 466, Issue 3, 2547 (2017)

[6] M. Milgrom, The Astrophysical Journal, 270, 365 (1983)

[7] M. Milgrom, Can. J. Phys. ,93, 126 (2015)

[8] S. McGaugh, Can. J. Phys., 93, 250 (2015)

[9] A. Einstein, Preuss. Akad. Wiss, Berlin (Math. Phys.), 142 (1917)

[10] J.D. Bekenstein, Phys. Rev. D7, 2333 (1973)

[11] S.W. Hawking, Comm. Math. Phys. 43, 199 (1975)

[12] W.G. Unruh, Phys. Rev. D 14, 870 (1976)

[13] http://www.scholarpedia.org/article/The_MOND_paradigm_of_modified_dynamics

[14] D. Hajdukovic, Astrophysics and Space Science, 334, vol.2, 215 (2011)

[15] L. Susskind, Inside blackholes, lecture on youtube

[16] Qing-yu Cai, Chang-pu Sun and LiYou, Nucl. Phys.B , 905 ,337 ( 2016)

[17] E.Roza, Results in Phys., vol 6, 149 (2016) 